\title{
Anemia e índices de atividade de doença em mulheres com artrite reumatoide
}

\author{
Anemia and disease activity indexes in women with rheumatoid arthritis
}

\author{
Thamiris Becker Scheffel1, Aline Defaveri do Prado², Henrique Luiz Staub³ , Inês Guimarães da Silveira ${ }^{3}$, Ana Lígia Bender ${ }^{4} \bowtie$ \\ ' Farmácia Clínica do Hospital São Lucas da Pontifícia Universidade Católica do Rio Grande do Sul (PUCRS). Porto Alegre, RS, Brasil. \\ 2 Serviço de Reumatologia do Hospital Nossa Senhora da Conceição. Porto Alegre, RS, Brasil. \\ ${ }^{3}$ Escola de Medicina da PUCRS. Porto Alegre, RS, Brasil. \\ ${ }^{4}$ Curso de Farmácia da Escola de Ciências da Saúde da PUCRS. Porto Alegre, RS, Brasil.
}

Como citar este artigo (How to cite this article):

Scheffel TB, Prado AD, Staub HL, Silveira IG, Bender AL. Anemia e índices de atividade de doença em mulheres com artrite reumatoide (Anemia and disease activity indexes in women with rheumatoid arthritis). Sci Med. 2018;28(2):ID28816. http://doi.org/10.15448/19806108.2018.2.28816

\section{RESUMO}

OBJETIVOS: Avaliar os índices de atividade de doença em pacientes do sexo feminino com artrite reumatoide, anêmicas e não anêmicas, correlacionando-os com os níveis de hemoglobina.

MÉTODOS: Um estudo transversal envolveu mulheres com artrite reumatoide classificadas em dois grupos: 1) Anêmicas (hemoglobina $<12 \mathrm{~g} / \mathrm{dL}$ ) e 2) Não anêmicas. A atividade da doença foi medida pelo Disease Activity Score (DAS28), utilizando-se marcadores inflamatórios distintos: velocidade de hemossedimentação (VHS) ou proteína C reativa (PCR). Esse escore também utiliza o número de articulações edemaciadas e dolorosas e a avaliação global da doença em escala analógica visual (EVA). Realizou-se também a avaliação da capacidade funcional pelo Health Assessment Questionaire (HAQ). A estatística utilizou os testes t de Student, Mann-Whitney, Wilcoxon, Fisher, likelihood ratio e correlação de Spearman. Foi considerado significativo $\mathrm{p}<0,05$.

RESULTADOS: Foram incluídas 24 pacientes, sendo oito do grupo Anêmicas e 16 do grupo Não anêmicas. Os grupos foram semelhantes quanto às características clínicas, demográficas e de tratamento, diferindo apenas em relação ao fator reumatoide, positivo em todas as participantes anêmicas e em 56,2\% das não anêmicas. O DAS28 VHS (mediana 6,05; intervalo interquartis [IIQ] 5,21-7,76), o DAS28 PCR (mediana 4,32; IIQ 3,98-5,92) e a EVA (mediana 66,50 mm, IIQ 54,75-80,50) foram significativamente maiores no grupo Anêmicas. O DAS28 VHS (-0,418) e a EVA $(-0,426)$ apresentaram correlação negativa significativa com o nível de hemoglobina. Os valores de DAS28 VHS e DAS28 PCR foram diferentes no mesmo grupo, mostrando discrepância na categorização da atividade da doença. No grupo Anêmicas, o valor de DAS28 VHS (mediana 6,05; IIQ 5,21-7,76) foi maior em relação ao DAS28 PCR (mediana 4,32; IIQ 3,98-5,92). Um aumento menos discrepante de DAS28 VHS (mediana 4,01; IIQ 3,05-5,68) comparado ao DAS28 PCR (mediana 3,06; IIQ 2,18-4,66) foi observado no grupo Não anêmicas. CONCLUSÕES: A anemia foi associada a piores índices de atividade de doença nas mulheres com artrite reumatoide, estando correlacionada com maior intensidade de dor e aumento do escore DAS28 VHS.

DESCRITORES: artrite reumatoide; doenças reumáticas; anemia

\section{ABSTRACT}

AIMS: To evaluate disease activity indexes in female patients with rheumatoid arthritis, Anemic and Non-anemic, correlating them with hemoglobin levels.

METHODS: A cross-sectional study involved women with rheumatoid arthritis classified into two groups: 1) Anemic (hemoglobin $<12 \mathrm{~g} / \mathrm{dL}$ ) and 2) Non-anemic. Disease activity was measured by Disease Activity Index (DAS28), using different inflammatory markers: Erythrocyte Sedimentation Rate (ESR) and C-reative Protein (CRP). This score also uses the number of swollen and painful joints and an overall assessment of the disease on Visual Analogue Scale (VAS). An assessment of functional capacity by the Health Assessment Questionnaire (HAQ) was also performed. The statistic used Student's t-Test, Mann-Whitney, Wilcoxon, Fisher, likelihood ratio and Spearman correlation tests. It was considered significant $\mathrm{p}<0,05$.

RESULTS: Twenty-four patients were included, eight patients in the Anemic group and 16 patients in the Non-anemic group. The groups were similar in terms of clinical, demographic and treatment characteristics, differing only in relation to rheumatoid factor, positive in all anemic participants and in 56,2\% of non anemic participants. DAS28 ESR (median 6,05; interquartile range [IQR] 5,21-7,76), DAS28 CRP (median 4,32; IQR 3,98-5,92) and VAS (median 66,50 mm; IQR 54,75-80,50) were significantly higher in Anemic group. DAS28 ESR (-0,418) and VAS $(-0,426)$ showed a significant negative correlation with hemoglobin level. DAS28 ESR and DAS28 CRP values were different in the same group, showing a discrepancy in the categorization of disease activity. In Anemic group, DAS28 ESR value (median 6,05; IQR 5,21-7,76) was higher in relation to DAS28 CRP (median 4,32; IQR 3,98-5,92). A less discrepant increase of DAS28 ESR (median 4,01; IQR 3,05-5,68) compared to DAS28 CRP (median 3,06; IQR 2,18-4,66) was observed in Non-anemic group.

CONCLUSIONS: Anemia was associated with worse disease activity indexes in women with rheumatoid arthritis, being correlated with greater pain intensity and increase of DAS28 ESR score.

KEYWORDS: rheumatoid arthritis; rheumatic diseases; anemia 
Abreviaturas: AR, artrite reumatoide; DAS28, Disease Activity Score; DMCD, Drogas Modificadoras do Curso da Doença; EVA, escala analógica visual; FR, fator reumatoide; HAQ, Health Assessment Questionaire; IIQ, intervalo interquartil; PCR, proteína $\mathrm{C}$ reativa; VHS, velocidade de hemossedimentação.

\section{INTRODUÇÃO}

A artrite reumatoide (AR) é uma doença autoimune inflamatória crônica que acomete cerca de $1 \%$ da população, sendo mais prevalente em mulheres de meia idade. Caracteriza-se por sinovite inflamatória, envolvendo principalmente as articulações periféricas, com potencial de dano ósseo e cartilaginoso, conforme sua progressão [1]. Além do característico comprometimento articular, o aumento de citocinas e de outros mediadores inflamatórios está relacionado ao desenvolvimento de complicações secundárias, tais como nódulos reumatoides, alterações hematológicas, comprometimento cardiovascular e comorbidades sistêmicas [2-4].

A AR tem grande impacto sobre a qualidade de vida dos pacientes, que sofrem com as deformidades articulares e a limitação funcional para exercer atividades cotidianas $[5,6]$. Em vista disso, um dos principais objetivos da terapia atual é controlar rigorosamente o processo inflamatório, a fim de reduzir a atividade da doença e suas implicações $[7,8]$.

Para avaliar a progressão da AR e sustentar decisões e ajustes terapêuticos recomenda-se o uso de instrumentos conhecidos como Índices Compostos de Atividade da Doença [9]. Dentre esses instrumentos, o Disease Activity Score (DAS28) é um dos mais empregados para categorizar a atividade da doença em uma escala contínua (remissão, atividade leve, moderada e alta) considerando o número de articulações dolorosas e edemaciadas (em um total de 28 articulações), a velocidade de hemossedimentação (VHS) ou o nível de proteína $\mathrm{C}$ reativa (PCR) e a avaliação global da doença [10]. Contudo, o uso de diferentes marcadores inflamatórios nesse instrumento tem gerado discordâncias na classificação da atividade da AR [11-13]. Embora o marcador VHS seja amplamente utilizado, ele pode sofrer influência de variáveis não relacionadas à inflamação, tais como idade, sexo, valores hematológicos e níveis séricos de fibrinogênio e de imunoglobulinas [14].

Alterações hematológicas, como a anemia, estão entre as principais manifestações extra articulares na AR, e exercem efeito direto sobre a VHS. Tal efeito pode influenciar o DAS28 e os demais marcadores relacionados à atividade da doença, causando discrepâncias nas condutas sobre a terapia e a avaliação prognóstica do paciente [15]. Portanto, o objetivo deste estudo foi avaliar os índices de atividade de doença em pacientes do sexo feminino com AR, anêmicas ou não anêmicas, correlacionando tais índices com os níveis de hemoglobina.

\section{MÉTODOS}

Foi realizado um estudo transversal envolvendo mulheres diagnosticadas com AR conforme os critérios do American College of Rheumatology [16], acompanhadas no Serviço de Reumatologia do Hospital São Lucas da Pontifícia Universidade Católica do Rio Grande do Sul (PUCRS). Este trabalho foi aprovado pelo Comitê de Ética em Pesquisa da PUCRS sob o Parecer Consubstanciado 425.110 e todas as participantes assinaram o Termo de Consentimento Livre e Esclarecido. Foram incluídas mulheres com idade superior a 18 anos e em tratamento exclusivo com Drogas Modificadoras do Curso da Doença (DMCD) sintéticas (metotrexato, hidroxicloroquina, sulfassalazina e leflunomida). Pacientes em tratamento com drogas biológicas associadas às DMCD sintéticas ou em uso isolado foram excluídas do estudo, devido à ação imune específica dessas drogas.

As participantes foram divididas em dois grupos, com base na sua concentração de hemoglobina: 1) Anêmicas e 2) Não anêmicas. A classificação seguiu os critérios para anemia da Organização Mundial da Saúde, que considera anêmicas mulheres com nível de hemoglobina inferior a $12,0 \mathrm{~g} / \mathrm{dL}$ [17]. Os dados demográficos, clínicos e de tratamento foram coletados dos prontuários médicos.

A atividade da doença foi avaliada pelo escore DAS28 conforme Fransen \& Riel [11], comparando-se dois marcadores inflamatórios distintos: VHS ou PCR. O escore DAS28 inclui a avaliação de 28 articulações (10 metacarpofalangeanas, 10 interfalangeanas proximais das mãos, dois punhos, dois cotovelos, dois ombros e dois joelhos) em relação à dor e ao edema, a quantificação dos marcadores inflamatórios VHS ou PCR e a avaliação global da doença medida em escala analógica visual (EVA), de zero (sem dor) a $100 \mathrm{~mm}$ (dor muito intensa) [10,18]. Considerou-se remissão da AR quando a pontuação do DAS28 foi inferior a 2,6, baixa atividade de 2,6 a 3,2 pontos, atividade moderada de 3,2 a 5,1 e atividade intensa acima de 5,1 pontos, sendo 10 a pontuação máxima do índice [10]. 
Os exames laboratoriais foram realizados no Laboratório Clínico do Hospital São Lucas da PUCRS. As quantificações dos marcadores inflamatórios VHS e PCR foram realizadas por fotometria capilar de fluxo no equipamento TEST1 THL - Alifax ${ }^{\circledR}$ e por química seca no equipamento VITROS Fusion 5.1 ${ }^{\circledR}$ (Ortho Clinical Diagnostics, Johnson \& Johnson), respectivamente.

Os parâmetros hematimétricos foram processados em analisador hematológico Sysmex XE-5000 ${ }^{\circledR}$. O fator reumatoide (FR) foi quantificado por turbidimetria em analisador VITROS Fusion 5.1 ${ }^{\circledR}$ (Ortho Clinical Diagnostics, Johnson \& Johnson), sendo considerados positivos valores superiores a $12 \mathrm{UI} / \mathrm{mL}$.

Para a avaliação da capacidade funcional utilizou-se como instrumento o questionário Health Assessment Questionaire (HAQ), aplicado na avaliação inicial por reumatologista treinado, com 20 questões divididas em oito componentes sobre as atividades de vida diária (vestir-se e arrumar-se, levantar-se, alimentar-se, caminhar, higiene, alcançar objetos, preensão e desempenho de atividade). Os valores de HAQ até 1 indicam limitação leve, superiores a 1 até 2 indicam limitação moderada e superiores a 2 até 3 , limitação grave [19].

A análise estatística foi realizada utilizando-se o programa SPSS Statistics para Windows, versão 17.0. Variáveis com distribuição normal foram apresentadas como média e desvio padrão, e comparações entre os grupos foram realizadas usando o Teste $t$ de Student. Variáveis ordinais ou quantitativas com distribuição não normal foram descritas como mediana e intervalo interquartis (IIQ, representando os valores dos percentis 25 e 75), e as comparações entre e intra grupos foram feitas usando os testes de Mann-Whitney, Wilcoxon ou Fisher. O teste de razão de verossimilhança (likelihood ratio) foi aplicado para as variáveis com distribuição não normal. Para pesquisa de correlação utilizou-se o teste de correlação de Spearman. Valores de $p$ iguais ou inferiores a 0,05 foram considerados significativos.

\section{RESULTADOS}

O estudo contou com a participação de 24 mulheres diagnosticadas com AR, sendo oito do grupo Anêmicas e 16 do grupo Não anêmicas. As características demográficas, clínicas e de tratamento das pacientes estão apresentadas na Tabela 1. Não houve diferença estatisticamente significativa em relação à caracterização demográfica, ao tempo médio de doença e ao tratamento farmacológico. Os grupos também foram semelhantes em relação à presença de comorbidades. O FR foi positivo em todos os casos do grupo Anêmicas, diferindo significativamente do grupo Não anêmicas, com $9(56,2 \%)$ casos positivos: likelihood ratio igual a 7,045.

Tabela 1. Características demográficas, clínicas e de tratamento de 24 pacientes com artrite reumatoide participantes do estudo.

\begin{tabular}{|c|c|c|c|}
\hline Característica & $\begin{array}{l}\text { Anêmicas } \\
\qquad(n=8)\end{array}$ & $\begin{array}{c}\text { Não anêmicas } \\
(n=16)\end{array}$ & Valor de $\mathrm{p}$ \\
\hline Idade (anos) - média \pm desvio padrão & $57,25 \pm 14,89$ & $57,56 \pm 11,84$ & $0,956^{*}$ \\
\hline Cor da pele branca - n (\%) & $6(75,0)$ & $14(87,5)$ & $0,350^{+}$ \\
\hline Tempo de doença (anos) - mediana (IIQ 25 e 75) & $14,0(6,25-29,0)$ & $9,5(5,0-16,0)$ & $0,417 \S$ \\
\hline Fator reumatoide positivo - $\mathrm{n}(\%)$ & $8(100)$ & $9(56,2)$ & $0,033^{+}$ \\
\hline Prednisona - n (\%) & $8(100)$ & $13(81,3)$ & $0,277^{+}$ \\
\hline Metotrexato - n (\%) & $5(62,5)$ & $13(81,3)$ & $0,302^{+}$ \\
\hline Leflunomida - n (\%) & $6(75,0)$ & $6(37,5)$ & $0,097^{+}$ \\
\hline Antimaláricos - n (\%) & $1(12,5)$ & $6(37,5)$ & $0,218^{+}$ \\
\hline Sulfassalazina $-\mathrm{n}(\%)$ & $1(12,5)$ & $N A^{* *}$ & $0,333^{+}$ \\
\hline Hipertensão - n (\%) & $7(87,5)$ & $11(68,7)$ & $0,319^{+}$ \\
\hline Diabetes Mellitus - n (\%) & $1(12,5)$ & $2(12,5)$ & $0,723^{+}$ \\
\hline Dislipidemia - n (\%) & $4(50,0)$ & $3(18,7)$ & $0,134^{+}$ \\
\hline Síndrome de Sjögren - n (\%) & NA & $2(12,5)$ & $0,435^{+}$ \\
\hline Osteoporose - n (\%) & $3(37,5)$ & $3(18,7)$ & $0,302^{+}$ \\
\hline Artrose $-\mathrm{n}(\%)$ & $4(50,0)$ & $7(43,7)$ & $0,556^{+}$ \\
\hline Obesidade - n (\%) & $5(62,5)$ & $8(50,0)$ & $0,444^{\dagger}$ \\
\hline Tabagismo - n (\%) & $1(12,5)$ & 0 & $0,333^{+}$ \\
\hline
\end{tabular}

Anêmicas: grupo com hemoglobina $<12 \mathrm{~g} / \mathrm{dL}$. Não anêmicas: grupo com hemoglobina $\geq 12 \mathrm{~g} / \mathrm{dL}$

* Teste t de Student; †Teste exato de Fisher; §Teste de Mann-Whitney. 
Tabela 2. Comparação dos parâmetros relacionados à atividade da artrite reumatoide entre os grupos em estudo.

\begin{tabular}{lccc}
\hline Parâmetro & $\begin{array}{c}\text { Anêmicas } \\
(\mathbf{n = 8})\end{array}$ & $\begin{array}{c}\text { Não anêmicas } \\
(\mathbf{n = 1 6 )}\end{array}$ & $\begin{array}{c}\text { Valor de } \mathbf{p}^{*} \\
\text { Articulações edemaciadas }\end{array}$ \\
$\begin{array}{l}\text { Articulações dolorosas } \\
\text { Disease Activity Score 28 VHS }\end{array}$ & $8,00(3,00-9,25)$ & $3,00(0,50-7,00)$ & 0,320 \\
Disease Activity Score 28 PCR & $6,05(5,75-26,50)$ & $2,50(1,25-11,75)$ & 0,136 \\
HAQ & $4,32(3,98-5,92)$ & $3,06(2,18-4,66)$ & $\mathbf{0 , 0 1 1}$ \\
EVA $(\mathrm{mm})$ & $1,94(1,44-2,62)$ & $1,12(0,19-1,84)$ & 0,070 \\
\hline
\end{tabular}

Os dados estão apresentados em mediana e intervalo interquartil (percentis 25 e 75).

Anêmicas: grupo com hemoglobina $<12 \mathrm{~g} / \mathrm{dL}$. Não anêmicas: grupo com hemoglobina $\geq 12 \mathrm{~g} / \mathrm{dL}$

VHS, velocidade de hemossedimentação; PCR, proteína C reativa; HAQ, Health Assessment Questionaire; EVA, Escala Visual Analógica.

* Teste de Mann-Whitney.

Os resultados da comparação da atividade de doença entre os grupos são mostrados na Tabela 2. Tanto o DAS28 quanto a EVA foram significativamente maiores nas pacientes anêmicas em relação ao grupo de pacientes com níveis normais de hemoglobina. Além disso, verificou-se diferença estatisticamente significativa entre DAS28 VHS e DAS28 PCR no grupo Anêmicas $(p=0,012)$ e no grupo Não anêmicas $(p=0,001)$, mostrando discrepância na categorização da atividade de doença dentro do mesmo grupo por diferentes testes.

Os resultados da análise de correlação entre o nível de hemoglobina e os parâmetros relacionados à atividade da AR estão descritos na Tabela 3. Observou-se correlação negativa significativa entre o nível de hemoglobina, o DAS28 VHS e a EVA.

Tabela 3. Correlação entre o nível de hemoglobina e os parâmetros relacionados à atividade da artrite reumatoide.

\begin{tabular}{lcc}
\hline Parâmetro & $\begin{array}{c}\text { Coeficiente de } \\
\text { correlação* }\end{array}$ & Valor de p \\
\hline Articulações edemaciadas & $-0,223$ & 0,295 \\
Articulações dolorosas & $-0,174$ & 0,417 \\
Disease Activity Score 28 VHS & $-0,418$ & $\mathbf{0 , 0 4 2}$ \\
Disease Activity Score 28 PCR & $-0,290$ & 0,169 \\
HAQ & $-0,216$ & 0,310 \\
EVA & $-0,426$ & $\mathbf{0 , 0 3 8}$ \\
\hline
\end{tabular}

VHS, velocidade de hemossedimentação; PCR, proteína $C$ reativa; HAQ, Health Assessment Questionaire; EVA, Escala Visual Analógica.

* Teste de correlação de Spearman.

\section{DISCUSSÃO}

A anemia tem se mostrado uma manifestação importante na AR, tanto por sua gravidade e alta prevalência em doenças reumáticas sistêmicas, quanto por sua influência negativa na qualidade de vida dos pacientes. Cerca de 33 a $60 \%$ dos indivíduos com doenças reumáticas sistêmicas podem ter anemia de doença crônica. Na maior parte das vezes, a anemia apresenta-se de forma leve ou moderada, normocítica e normocrômica [20].

Sabe-se que a anemia na doença inflamatória sistêmica é imunomediada. Citocinas e células do sistema reticulo-endotelial (SRE) induzem alterações que interferem em diferentes vias da eritropoese. A homeostase patológica do ferro induzida pela hepcidina é um dos mecanismos etiológicos mais evidentes, embora a diminuição da proliferação dos progenitores eritroides, a redução da atividade biológica da eritropoietina e o encurtamento da meia vida dos eritrócitos também possam ser citados [20]. Além disso, outros mecanismos, como deficiências vitamínicas ou efeitos adversos de medicamentos, podem contribuir ou agravar a anemia [21]. Apesar da relevância dessa comorbidade, existem ainda algumas lacunas na compreensão das suas consequências em pacientes com AR.

Neste estudo, a anemia interferiu significativamente nos índices de atividade de doença nas pacientes artríticas. Valores maiores de DAS28 PCR e DAS28 VHS foram observados no grupo de pacientes anêmicas quando comparado ao grupo de pacientes com níveis normais de hemoglobina.

Os dois grupos foram semelhantes, sem diferenças significativas entre o tempo de doença, o tratamento farmacológico e as comorbidades existentes. No entanto, todas as pacientes do grupo Anêmicas apresentaram FR positivo, com um likelihood ratio igual a 7,045, indicando uma chance maior de um paciente artrítico com FR positivo ter anemia em relação ao paciente com FR negativo. A associação do FR com manifestações extra-articulares já é 
bem conhecida [4]. Moura et al. encontraram FR positivo em aproximadamente $70 \%$ dos pacientes com manifestação extra-articular, evidenciando a associação deste marcador com as complicações da AR [4]. Diante disso, o resultado encontrado neste estudo sobre o FR pode justificar o índice mais elevado de DAS28 PCR observado no grupo Anêmicas em relação ao grupo Não anêmicas. Conforme alguns autores já demonstraram, existe uma associação direta entre os níveis de PCR e FR [22,23].

Do mesmo modo, as pacentes anêmicas também apresentaram DAS28 VHS maior em relação às pacientes não anêmicas. Isto porque a redução do nível de hemoglobina e o baixo hematócrito aceleraram a velocidade de sedimentação das hemácias, aumentando o valor de DAS28 VHS [20]. Este escore de atividade de doença é amplamente empregado na avaliação clínica, principalmente em função do custo reduzido do marcador VHS em relação à PCR quantitativa, porém, alguns pontos precisam ser considerados. A VHS sofre forte interferência de variáveis não relacionadas ao processo inflamatório e reflete a atividade inflamatória de algumas semanas, apresentando maior sensibilidade para alterações lentas [22].

Um achado importante foi a discrepância nos resultados do DAS28 conforme a utilização dos marcadores VHS ou PCR. Tanto no grupo Anêmicas, quanto no grupo Não anêmicas, o valor de DAS28 VHS mostrou-se significativamente maior do que DAS28 PCR. O impacto clínico desta discrepância está relacionado à diferença na classificação da atividade da AR e na definição do real estado do paciente. No grupo Não anêmicas, as pacientes passaram de baixa atividade com o DAS28 PCR para moderada atividade de doença com o DAS28 VHS, enquanto que, mais gravemente, no grupo Anêmicas a mudança foi da categoria de moderada atividade para intensa atividade de doença.

Da mesma forma, outros estudos apresentaram achados de subestimação da atividade da AR relacionada ao escore DAS28 PCR [11-14,24]. A PCR não sofre a mesma influência dos fatores citados sobre a VHS e reflete mudanças de curto prazo, sendo mais sensível para alterações rápidas na atividade de doença. Existe também uma boa correlação da PCR com a terapêutica e a progressão radiológica [22].

Os escores de atividade de doença são importantes no tratamento da AR, pois auxiliam na definição do real estado do paciente, balizando as decisões e os ajustes terapêuticos. A definição precisa e correta de alta atividade de doença, por exemplo, é importante para se definir o início da terapia com agentes biológicos e, também por razões prognósticas, já que a possibilidade de uma progressão acelerada é maior em pacientes com atividade mais alta. O contrário também é verdadeiro: é preciso ter segurança para se definir um paciente com baixa atividade ou remissão da doença, evitando que este deixe de receber um tratamento necessário [13].

A relação entre inflamação e anemia está bem estabelecida. Dependendo do grau da anemia, esta pode afetar negativamente o desempenho cardiovascular, a funcionalidade física e a qualidade de vida do paciente [20]. Em 2014, Ganna [15] e Möller et al. [25] demonstraram que baixos níveis de hemoglobina podem estar relacionados a um pior desfecho na AR, em razão da associação da anemia com o escore de atividade de doença e, consideravelmente, com os parâmetros de avaliação da capacidade funcional e da dor relatados pelo paciente.

Em se tratando de uma doença em que o controle da dor é considerado como parte do tratamento de remitência, é importante valorizar as avaliações feitas pelo paciente para que os resultados terapêuticos sejam analisados [26]. Em nosso estudo as pacientes anêmicas relataram uma intensidade de dor muito maior em relação às pacientes com níveis normais de hemoglobina, mostrando uma forte correlação entre a anemia e a EVA. Outros estudos também já relataram essa associação [26-28].

Inúmeros fatores podem influenciar a avaliação que o paciente faz da própria doença. Sabe-se que a AR é impactante para a qualidade de vida do indivíduo que sofre com a intensidade do processo inflamatório, o dano estrutural, a terapia medicamentosa, a repercussão emocional e as comorbidades existentes, tais como a anemia [28]. Apesar de nosso estudo não ter demonstrado diferença significativa relacionada ao HAQ, possivelmente em função do número reduzido de participantes, as pacientes anêmicas relataram maiores dificuldades em tarefas simples do dia-adia, mostrando uma limitação moderada. Conforme Medeiros et al. [13], a fadiga causada pela anemia e as limitações físicas podem afetar diretamente a avaliação da capacidade funcional.

A qualidade de vida é de fundamental importância e tem sido avaliada pelo uso de ferramentas como o HAQ. Porém, de acordo com Mota et al. [29], alguns pacientes têm relatado que os parâmetros de avaliação da qualidade de vida usados de forma independente não abordam aspectos importantes como fadiga e perturbação do sono, que certamente afetam a qualidade de vida [29].

Entre os fatores limitantes deste estudo, salientamos o baixo número amostral e o número reduzido de 
pacientes anêmicas, o que pode ter prejudicado a interpretação dos dados estatísticos. No entanto, as limitações não impediram de mostrar que a anemia pode estar associada a piores resultados relacionados aos índices de atividade de doença em pacientes com AR.

As pacientes anêmicas apresentaram valores de DAS28 e EVA significativamente maiores em comparação àquelas com níveis normais de hemoglobina. Além disso, a anemia correlacionou-se com maior intensidade da dor e aumento do escore DAS28 VHS. O problema da interferência da anemia sobre o DAS28 VHS é a discrepância observada em relação ao DAS28 PCR, que pode gerar uma sub ou superestimação da atividade da doença e impactar diretamente na classificação do estado do paciente. Dessa forma, mais estudos são necessários para estabelecer o marcador inflamatório que mais precisamente indique o real estado do paciente, levando-se em consideração fatores demográficos e clínicos que possam interferir na avaliação da atividade da doença.

\section{NOTAS}

Apoio financeiro

Este estudo recebeu apoio financeiro do Fundo de Apoio à Pesquisa da Sociedade de Reumatologia do Rio Grande do Sul.

Declaração de conflito de interesses

Os autores declaram não haver conflitos de interesses relevantes ao conteúdo deste estudo.

\section{Contribuições dos autores}

Todos os autores fizeram contribuições substanciais para concepção, ou delineamento, ou aquisição, ou análise ou interpretação de dados; e redação do trabalho ou revisão crítica; e aprovação final da versão para publicação.

Disponibilidade dos dados

Os autores declaram ter tido acesso a todos os dados obtidos e assumem completa responsabilidade pela integridade destes resultados.

\section{REFERÊNCIAS}

1. Smolen JS, Aletaha D, Mcinnes IB. Rheumatoid arthritis. Lancet. 2016;388:2023-38. https://doi.org/10.1016/S01406736(16)30173-8

2. Burmester GR, Feist E, Dörner T. Emerging cell and cytokine targets in rheumatoid arthritis. Nat Rev Rheumatol. 2014;10:77-88. https://doi.org/10.1038/nrrheum.2013.168

3. Prado AD, Bisi MC, Piovesan DM, Bredemeier M, Batista TS, Petersen L, Bauerc ME, Silveira IG, Mendonça JA, Staub HL. Ultrasound power Doppler synovitis is associated with plasma IL-6 in established rheumatoid arthritis. Cytokine. 2016;83:27-32. https://doi.org/10.1016/j.cyto.2016.03.014

4. Moura MC, Zakszewski PTS, Silva MBG, Skare TL. Perfil dos pacientes com manifestações extra-articulares de artrite reumatoide de um serviço ambulatorial em Curitiba, Sul do Brasil. Rev Bras Reumatol. 2012;52(5):679-94. https://doi. org/10.1590/S0482-50042012000500004

5. Roma I, Almeida ML, Mansano NS, Viani GA, Assis MR, Barbosa PMK. Quality of life in adults and elderly patients with rheumatoid arthritis. Rev Bras Reumatol. 2014;54(4):279-86. https://doi.org/10.1016/j.rbr.2014.03.025

6. Marques WV, Cruz VA, Rego J, Silva NA. The impact of comorbidities on the physical function in patients with rheumatoid arthritis. Rev Bras Reumatol. 2016;56(1):14-21. https://doi.org/10.1016/j.rbr.2015.01.009

7. Singh JA, Saag KG, Bridges SL, Akl EA, Bannuru RR, Sullivan MC, Vaysbrot E, Mcnaughton C, Osani M, Shmerling RH, Curtis JR, Furst DE, Parks D, Kavanaugh A, O'dell J, King C, Leong A, Matteson EL, Schousboe JT, Drevlow B, Ginsberg S, Grober J, St.clair W, Tindall E, Miller AS, Mcalindon T. 2015 American College of Rheumatology Guideline for the Treatment of Rheumatoid Arthritis. Arthritis Rheumatol. 2016;68(1):1-26. https://doi.org/10.1002/art.39480

8. Stoffer MA, Schoels MM, Smolen JS, Aletaha D, Reedveld FCB, Burmester G, Bykerk V, Dougados M, Emery P, Haraoui B, Gomez-Reino J, Kvien TK, Nash P, Navarro-Compán V, Scholte-Voshaar M, van Vollenhoven R, van der Heijde D, Stamm TA. Evidence for treating rheumatoid arthritis to target: results of a systematic literature search update. Ann Rheum Dis. 2016;75:16-22. https://doi.org/10.1136/annrheumdis-2015-207526

9. Da Mota L, Cruz B, Brenol C, Pereira I, Rezende-Fronza L, Bertolo M. 2012 Brazilian Society of Rheumatology Consensus for the treatment of rheumatoid arthritis. Rev Bras Reumatol. 2012;52:152-74. http://doi.org/10.1590/S048250042012000200002

10. Fransen J, Riel PL. The disease activity score and the EULAR response criteria. Rheum Dis Clin North Am. 2009;35(4): 745-57. https://doi.org/10.1016/j.rdc.2009.10.001

11. Inoue E, Yamanaka H, Hara M, Tomatsu T, Kamatani N. Comparison of Disease Activity Score DAS28-erythrocyte sedimentation rate and DAS28-C-reactive protein threshold values. Ann Rheum Dis. 2007;66:407-9. https://doi. org/10.1136/ard.2006.054205

12. Belmonte M. Comparacion de la efectividad clínica y los umbrales de corte para el cálculo del DAS28 basado en VSG vs. PCR. Rev Sociedad Val Reuma 2011;4(1):1-3. 
13. Medeiros MMC, Oliveira BMGB, Cerqueira JVM, Quixada RTS, Oliveira IMX. Correlation of rheumatoid arthritis activity indexes (Disease Activity Score 28 measured with ESR and CRP, Simplified Disease Activity Index and Clinical Disease Activity Index) and agreement of disease activity states with various CUT-off points in a Northeastern Brazilian population. Rev Bras Reumatol. 2015;55(6):477-84. https://doi.org/10.1016/j.rbr.2014.12.005

14. Castrejón I, Ortiz AM, García-Vicu-a R, Lopez-Bote JP, Humbría A, Carmona L, Gonzalez-Alvaro I. Are the C-reactive protein values and erythrocyte sedimentation rate equivalent when estimating the 28 -joint disease activity score in rheumatoid arthritis? Clin Exp Rheumatol. 2008;26:769-75.

15. Ganna S. The relationship between hemoglobin level and disease activity in patients with rheumatoid arthritis. Rev Bras Reumatol. 2014;54(6):437-40. https://doi.org/10.1016/j.rbre.2014.06.003

16. Aletaha D, Neogi T, Silman AJ, Funovits J, Felson DT, Bingham CO, Birnbaum NS, Burmester GR, Bykerk VP, Cohen MD, Combe B, Costenbader KH, Dougados M, Emery P, Ferraccioli G, Hazes JM, Hobbs K, Huizinga TW, Kavanaugh A, Kay J, Kvien TK, Laing T, Mease P, Ménard HA, Moreland LW, Naden RL, Pincus T, Smolen JS, Stanislawska-Biernat E, Symmons D, Tak PP, Upchurch KS, Vencovsky J, Wolfe F, Hawker G. 2010 Rheumatoid Arthritis Classification Criteria: An American College of Rheumatology/European League Against Rheumatism Collaborative Initiative. Arthritis Rheumatol. 2010;62(9):2569-81. https://doi.org/10.1002/art.27584

17. World Health Organization. Haemoglobin concentrations for the diagnosis of anaemia and assessment of severity [internet]. 2011 [cited 2016 Nov 20]. Available from: http://www.who.int/vmnis/indicators/haemoglobin.pdf

18. Dansie EJ, Turk DC. Assessment of patients with chronic pain. Br J Anaesth. 2013;111(1):19-25. https://doi.org/10.1093/ bja/aet124

19. Maska L, Anderson J, Michaud K. Measures of functional status and quality of life in rheumatoid arthritis: Health Assessment Questionnaire Disability Index (HAQ), Modified Health Assessment Questionnaire (MHAQ), Multidimensional Health Assessment Questionnaire (MDHAQ), Health Assessment. Arthritis Care Res. 2011;63(11):S4-13. https://doi.org/10.1002/ acr. 20620

20. Weiss G, Schett G. Anaemia in inflammatory rheumatic diseases. Nat Rev Rheumatol. 2013;9(4):205-15. https://doi. org/10.1038/nrrheum.2012.183

21. Wilson A, Yu HT, Goodnough LT, Nissenson AR. Prevalence and outcomes of anemia in rheumatoid arthritis: a systematic review of the literature. Am Med J. 2004; 116(7):50S-57S. https://doi.org/10.1016/j.amjmed.2003.12.012

22. Neto NSR, Carvalho JF. O uso de provas de atividade inflamatória em reumatologia. Rev Bras Reumatol. 2009;49(4): 413-30. https://doi.org/10.1590/S0482-50042009000400008

23. Song YW, Kang EH. The pathogenic role of rheumatoid factor in rheumatoid arthritis. Int J Clin Rheumtol. 2010;5(6): 651-8. https://doi.org/10.2217/ijr.10.62

24. Tamhane A, Redden DT, McGwin GJr, Brown EE, Westfall AO, Reynolds RJ, Hughes LB, Conn DL, Callahan LF, Jonas BL, Smith EA, Brasington RD Jr, Moreland LW, Bridges SL Jr. Comparison of the disease activity score using erythrocyte sedimentation rate and C-reactive protein in African Americans with rheumatoid arthritis. J Rheumatol. 2013;40(11):1812-22. https://doi.org/10.3899/jrheum.121225

25. Möller B, Scherer A, Förger F, Villiger PM, Finckh A. Anaemia may add information to standardised disease activity assessment to predict radiographic damage in rheumatoid arthritis: a prospective cohort study. Ann Rheum Dis. 2014;73:691-6. https://doi.org/10.1136/annrheumdis-2012-202709

26. Corbacho MI, Dapueto JJ. Avaliação da capacidade funcional e da qualidade de vida de pacientes com artrite reumatoide. Rev Bras Reumatol. 2010;50(1):31-43. https://doi.org/10.1590/S0482-50042010000100004

27. Borah DJ, Iqbal F. Anemia in recent onset rheumatoid arthritis. JK Science 2007;9(3):120-2.

28. Furst DE, Chang H, Greenberg JD, Ranganath VK, Reed G, Ozturk ZE, Kremer JM. Prevalence of low hemoglobin levels and associations with other disease parameters in rheumatoid arthritis patients: Evidence from the CORRONA registry. Clin Exp Rheumatol. 2009;27:560-6.

29. Mota LM, Laurindo IM, de Carvalho JF, dos Santos-Neto LL. Prognostic evaluation of early rheumatoid arthritis. Swiss Med Wkly. 2010;140:w13100. https://doi.org/10.4414/smw.2010.13100 C 